\title{
Some aspects of Portevin-Le Chatelier plastic instabilities investigated by infrared pyrometry
}

\author{
N. Ranc*, D. Wagner \\ L.E.E.E., E.A.387, Université de Paris X Nanterre, 1, Chemin Desvallières, 92410 Ville d'Avray, France
}

\begin{abstract}
The deformation heterogeneity related to the Portevin-Le Chatelier effect has been studied for aluminium-copper alloy during a tensile test at ambient temperature. Plastic deformation is accompanied by dissipation of mechanical energy into heat. To observe the localization of deformation in bands, we used a pyrometer coupled to an infrared camera. This device serves to visualize the formation of the deformation bands and to quantify apparent velocity. From these thermal data, we can also determine the geometrical characteristics of the bands: orientation, bandwidth, and space between two bands. The aperture time $(1500 \mu \mathrm{s})$ and the acquisition frequency of the camera $(60 \mathrm{~Hz})$ allow the bandformation time and the middle-band time to be estimated. The measurement of the variation in temperature during the appearance of a band is used to quantify the plastic deformation in the band.
\end{abstract}

Keywords: Portevin-Le Chatelier effect; Dynamic strain aging; Plastic instability; Infrared pyrometry

\section{Introduction}

Strain aging processes are common in alloys containing solutes that are pinned by dislocations. Two types of strain aging can be characterized: static strain aging (SSA) [1,2] where the aging process takes place after straining and dynamic strain aging (DSA) [2-5] where aging is sufficiently rapid to occur during straining. In both cases, there is an increase in flow stress and work hardening rate and a decrease in ductility (elongation, reduction in area and fracture toughness) compared with the values expected in the absence of strain aging effects. The underlying physical models offered to interpret the experimental results are not well established. According to the review of models, reported by Kubin and Estrin [2,6], and Neuhäuser and Schwink [7], basic interpretations are generally accepted; the origin of these phenomena is related to the presence of solute atoms, which are able to diffuse over short distances and to pin mobile dislocations. In DSA a kind of resonance phenomenon occurs when the

\footnotetext{
* Corresponding author. Tel.: +331470970 13; fax: +33147091645. E-mail address: nicolas.ranc@u-paris10.fr (N. Ranc).
}

mobility of the solute species is of the same order as the dislocation velocity. Thus, depending on the composition and the thermo-mechanical treatment of the materials, the experimental conditions (temperature range, strain rate) required observing these interactions between mobile dislocations and diffusing atoms could vary considerably. In the early models [8] dislocations were assumed to move in a viscous manner while in more recent models [9-12] they were assumed to move in a jerky manner. Then the motion of dislocations is due to thermally activated jumps through localized obstacles and is characterized by a waiting time between jumps. During this waiting time, when the dislocations are temporarily stopped from obstacles, solute atoms diffuse to the dislocations and increase the strength of these obstacles.

Both types of strain aging can also give a rise in nonhomogeneous plastic deformation. Static strain aging results in a return of the Lüders strain phenomenon and dynamic strain aging produces a variety of types of non-homogeneous deformation called Portevin-Le Chatelier (PLC) effect. Within the range of existence of DSA, the strain rate sensitivity $S=\partial \sigma / \partial \ln \dot{\varepsilon}$ may become negative. In polycrystals subjected to DSA, deformed with a constant imposed strain 
rate, falling in the negative strain rate sensitivity range, PLC bands occur. On the stress-strain curve, these features produce serrated yielding. On a hard machine, three main types of serrated curves are commonly distinguished [7,13-19]. Type A serrations, which rise above the general level of the stress-strain curve, are associated with repetitive continuous propagation of deformation bands nucleated at certain location. These bands are often located at the specimen extremity and move along the specimen. Type B serrations oscillate about the general stress-strain curve and occur in rapid succession of localized deformation bands. Type $\mathrm{C}$ serrations fall below the general level of the stress-strain curve and occur when localized deformation bands appear in a spatially non correlated manner. Like in the case of type B, each stress drop reflects the formation of a single localized band. These types correspond to various modes of strain localization, which are correlated to external parameters (strain, strain rate, temperature, tensile machine stiffness, geometry sample, surface roughness of the sample) and internal parameters (composition of the alloy, crystal lattice, type and content of solute, single crystal or polycrystal, mobile dislocation density, obstacle type (forest, precipitates, ...) and grain size). The influence of the external parameters on the PLC effect was evaluated in experiments and theoretically. When the strain rate increases (waiting time of dislocations decreases) or the temperature decreases (mobility of solute decreases) the strain localization evolves from type $\mathrm{C}$ to type $\mathrm{B}$ and to type A. As a general trend, serrations occur after a certain amount of strain $\varepsilon_{\mathrm{c}}$ [20-22]. The critical strain for the onset of serrated yield normally increases with increasing strain rate and decreases with increasing temperature, but the inverse behaviour can occur. In some cases, a critical strain has also been observed with the disappearance of serrations $\varepsilon_{\mathrm{c}}$ [6].

Until now, experimental techniques used are video recording, extensometry by gauge length measurement, laser extensometry [7] and more recently acoustic emission [23] and laser speckle technique [24]. One of the most important difficulties in the study of the band propagation is the dynamic feature of the phenomenon. The plastic deformation of metals is accompanied by dissipation of the major part of the mechanical energy into heat. This dissipation generally results in an increase in the material temperature. In our study, we choose to measure the temperature fields on the surface of a specimen subjected to a tensile test. Because the PLC effect results from plastic deformation, this pyrometry technique enables us to survey the dynamic aspect of the phenomenon by visualising the appearance and the evolution of PLC bands, also to specify their geometrical characteristics as the band-widths and their orientation of which this phenomenon results. From the measurement of the temperature fields at various moments, the solution of the heat transfer equation obtains the cartography of the dissipated powers. If we know the expression of the dissipated power according to the stress and the plastic strain, we can determine the plastic strain field on the surface of the specimen. Thermography is also an experimental technique, which is able to study qualitatively and quantitatively the PLC effect.

After a description of our experimental device in Section 2, we will present the results obtained and the methods used for analysis. In Section 4, these results will be discussed and compared with those available in the literature.

\section{Experimental device}

\subsection{Materials}

The material used in this study is a $4 \%$ copper-aluminium alloy, heat treated at $500{ }^{\circ} \mathrm{C}$ for $30 \mathrm{~min}$ and then water quenched. Immediately after quench, the copper content in substitutional solid solution is important and this alloy is sensitive to DSA in a temperature range around $20^{\circ} \mathrm{C}$. The average grain size of the alloy was about $17 \mu \mathrm{m}$.

\subsection{Traction device}

Immediately after quench, tensile tests were carried out on a screw driven machine at the ambient temperature with a strain rate of $7.7 \times 10^{-3} \mathrm{~s}^{-1}$. Prismatic specimens of $12 \mathrm{~mm}$ wide, $3 \mathrm{~mm}$ thickness and $60 \mathrm{~mm}$ length were machined and covered with a black paint. The specimen's surface was in the as-rolled condition. The combined stiffness of the system "specimen-machine" was approximately $1.1 \times 10^{7} \mathrm{~N} / \mathrm{m}$. Conventional stress-nominal strain curves were plotted.

\subsection{Temperature measurements}

To determine the temperature field on the surface of the specimen, we chose a measurement technique by pyrometry. The advantages of this method are numerous. First, it is a non intrusive measurement technique, which does not disturb the temperature field in the specimen. It also obtains relatively short measurement times, about one millisecond in our study. Another advantage of pyrometry is to enable the visualization of temperature cartography with very good space resolutions. In our case, one measurement zone corresponds to an area of $0.4 \mathrm{~mm} \times 0.4 \mathrm{~mm}$ on the specimen surface. Pyrometry has been already used in mechanics for the study of other phenomena [25-29].

The design of a pyrometer requires choosing a spectral range, which is linked to the measured temperature and spectral sensitivity of the selected detector. For the Al-Cu alloy, DSA occurs at ambient temperature. During the tensile test, the temperature varies between the ambient and approximately $40^{\circ} \mathrm{C}$. The spectral range of the radiation emitted by the specimen is in the mid infrared domain associated to the mercury cadmium telluride detector (MCT detector). Also, in our study, we used an infrared camera made up of a matrix of $320 \times 240$ detectors. The aperture time is fixed at $1500 \mu \mathrm{s}$. The refresh frequency of the camera is of $60 \mathrm{~Hz}$, which corresponds to the duration between two pictures of $16.7 \mathrm{~ms}$. The 
weak noise of the camera (about $20 \mathrm{mK}$ ) detects weak variations of temperature. To focus the maximum energy emitted by the specimen, we use an objective with a $50 \mathrm{~mm}$ focal length.

The pyrometer is calibrated on a blackbody reference in order to establish the relation between the detector's output signals and the temperature. The sources of error associated with pyrometry are often related to uncertainties on the emmisivity of the surface. In this study, to eliminate this problem, we covered the specimen surface with a strongly emissive black painting. The thickness of the coat of paint is sufficiently fine so as not to disturb the temperature measurement of the specimen.

\section{Results and analysis methods}

\subsection{Results of mechanical and thermal measurements}

Fig. 1 shows the conventional stress-strain curve and the temperature measured on three points of the sample noted A, $\mathrm{B}$ and $\mathrm{C}$ and spaced $15 \mathrm{~mm}$. At the beginning of the tensile test, in the elastic range, we noted a temperature reduction of approximately $0.3^{\circ} \mathrm{C}$ due to the thermoelastic effect [30]. On the contrary, during the plastic deformation the temperature increases by several tens of degrees. This phenomenon is explained by the glide mechanism of the dislocations [31]. The stress-strain curve presents characteristic serrations of the PLC effect. This feature is composed of a first serration marked (a) followed by smaller serrations between (a) and (b). Smaller serrations are of type B, and evolve to type $\mathrm{C}$ at the end of the test. During the tensile test, we observed an in- crease in the average size of the stress drops. The temperature field on the surface specimen were recorded throughout the test. An example is given in Fig. 2 for deformations varying between 0.095 and 0.114 .

\subsection{Plastic deformations determination}

The temperature field in the specimen obeys the following heat transfer equation:

$\rho C_{\mathrm{p}} \frac{\partial T}{\partial t}=d_{\mathrm{int}}+\lambda \Delta T$

with $\rho$ the density, $C_{\mathrm{p}}$ the heat capacity and $\lambda$ the thermal conductivity.

The terms $\rho C_{\mathrm{p}} \dot{T}$ and $d_{\text {int }}$ of Eq. (1) correspond respectively to the calorific power stored in the material and to the power dissipated into heat per unit of volume. The Laplacian term $\lambda \Delta T$ corresponds to the phenomenon of heat conduction. The measurement of the temperature at various moments estimates the terms $\rho C_{\mathrm{p}} \dot{T}$ and $\lambda \Delta T$ and identifies the dissipated power $d_{\text {int }}$. A solution of this problem was proposed by Chrysochoos et al. [25]. In the case of thermoplasticity, we can express the dissipated power in the following form [31]:

$d_{\text {int }}=\beta \sigma: \frac{\partial \varepsilon_{\mathrm{p}}}{\partial t}$

with $\sigma$ the stress tensor, $\varepsilon_{\mathrm{p}}$ the plastic strain tensor, $\beta$ the Taylor Quiney coefficient and the symbol: the contract product.

By using this expression for $d_{\text {int }}$ in Eq. (1), we obtain:

$$
\rho C_{\mathrm{p}} \frac{\partial T}{\partial t}=\beta \sigma: \frac{\partial \varepsilon_{\mathrm{p}}}{\partial t}+\lambda \Delta T
$$

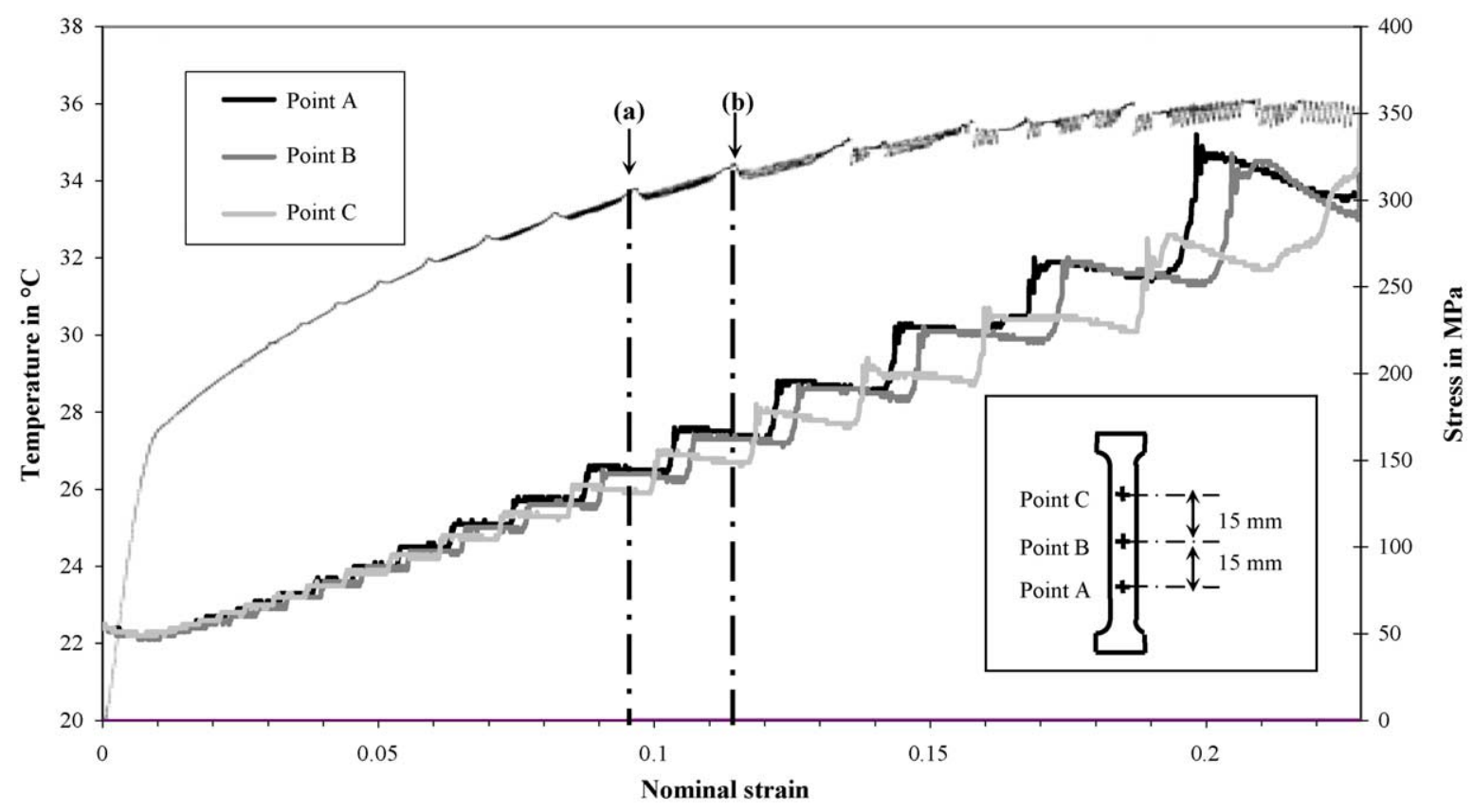

Fig. 1. Stress-strain curve and temperature evolution. 


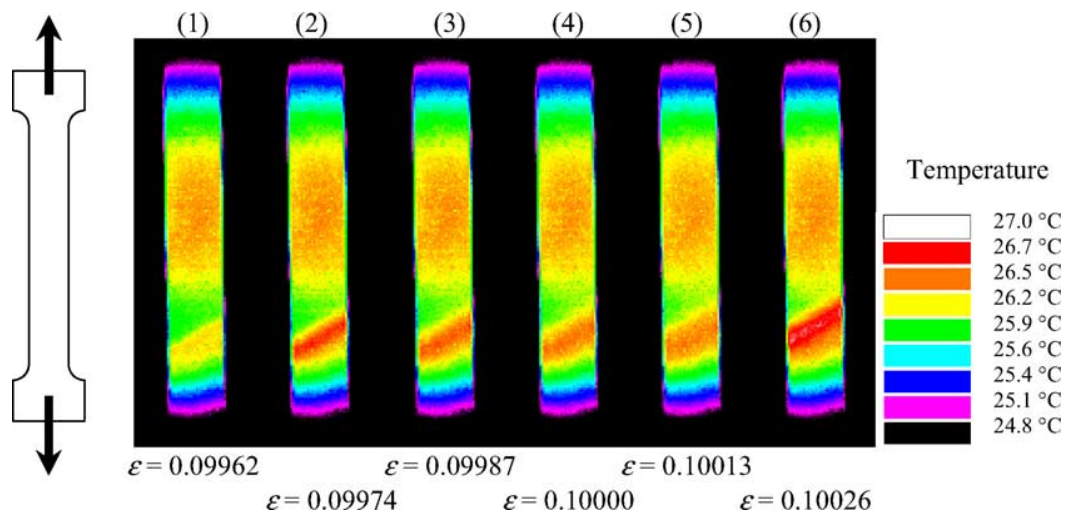

Fig. 2. Consecutive thermographs of traction specimen.

Assuming the process is adiabatic, the previous equation is reduced to:

$\rho C_{\mathrm{p}} \mathrm{d} T=\beta \sigma: \mathrm{d} \varepsilon_{\mathrm{p}}$

For a tensile test, we suppose that all the components of the stress tensor are zero except the normal component along the traction axis noted $\sigma$ and a relation of proportionality can be established between the temperature variation and the variation of plastic strain along the traction axis noted $\mathrm{d} \varepsilon_{\mathrm{p}}$ :

$\mathrm{d} \varepsilon_{\mathrm{p}}=\frac{\rho C_{\mathrm{p}}}{\beta \sigma} \mathrm{d} T$

If we measure the temperature variations with the infrared camera and if we know the stress from the strain-stress curve, we can determine the plastic strain variations.

\section{Discussion}

\subsection{Observations of the PLC band-formation and propagation}

The relationship between the stress-strain curve and the temperature field recordings enables precise macroscopic observations of the PLC bands formation. The temperature recordings at the A, B, C points (Fig. 1) show a sudden increase in the temperature when a band goes in front of the measurement point. We can conclude that there is an increase of the plastic deformation at the point. Fig. 2 shows six consecutive frames for deformation around 0.1 . We can observe a significant increase of the temperature between thermographies 1 and 2 and thermographies 5 and 6, which corresponds to a PLC band-formation. On the contrary, we can notice a slight decrease of the temperature between thermographies 2 and 5 .

Fig. 3 shows the formation of a new single band, which corresponds to one serration or one stress drop. All the frames on Fig. 3 are taken in the domain between (a) and (b). Each type (a) or (b) serration corresponds to the creation of a band at the same extremity of the sample (frames 1 and 26 in Fig. 3).
Both serrations probably result from the specimen geometry and our attention focuses on the pure type B serrations between (a) and (b).

At each point $\mathrm{A}$ or $\mathrm{B}$ or $\mathrm{C}$, when the band disappeared the temperature slightly decreased due to the heat diffusion in the sample around the point. This heat diffusion is equally visible between frames 2 and 5 in Fig. 2, near the PLC band previously formed. Frame 1 in Fig. 2 corresponds to a thermography just before the band-formation seen in frame 2 . Fig. 4 represents the temperature variation occurring before and after the formation of a PLC band (for instance between frame 1 and 2 of Fig. 2). As we saw in Section 3.2, this temperature variation is proportional to the increment of the plastic deformation (Eq. (5)). Our measurement device does not detects temperature variation lower than $40 \mathrm{mK}$ (for a signal to noise ratio equal to 2), which corresponds to a plastic deformation of $0.05 \%$. We can conclude that during the formation of the band the plastic deformation outside the band is lower than $0.05 \%$.

Fig. 4 shows clearly the organisation of the PLC bands formation. Thus, as expected, for these type B serrations the propagation of the localized bands in the axial direction is discontinuous but regular (Fig. 2). Although the band propagation is not continuous, an apparent velocity of the bands can be calculated from the measurement of the time difference between the A, B, C, points in Fig. 1. In Figs. 5 and 6, the apparent velocity is shown as a function of total strain and stress during the test. The apparent band velocities vary from more than $100 \mathrm{~mm} \mathrm{~s}^{-1}$ at the beginning of the test to $20 \mathrm{~mm} \mathrm{~s}^{-1}$ at the end of the test. The band velocity decreases with increasing strain and stress. The evolution of the apparent velocity is linear versus the stress. McCormick [17] and Cuddy and Leslie [14] on respectively an Al-Mg-Si alloy and an $\mathrm{Fe}-\mathrm{Ti}-\mathrm{Si}$ alloy have reported the same trend for type A serrations, but their values of velocity bands were lower (2-30 $\mathrm{mm} \mathrm{s}^{-1}$ for [17], 0.15-0.5 $\mathrm{mm} \mathrm{s}^{-1}$ for [14]). Any apparent velocity bands for type $\mathrm{B}$ or type $\mathrm{C}$ values have been reported in the literature.

On our alloy, the creation of the bands displayed by the non monotonic temperature evolution versus nominal defor- 

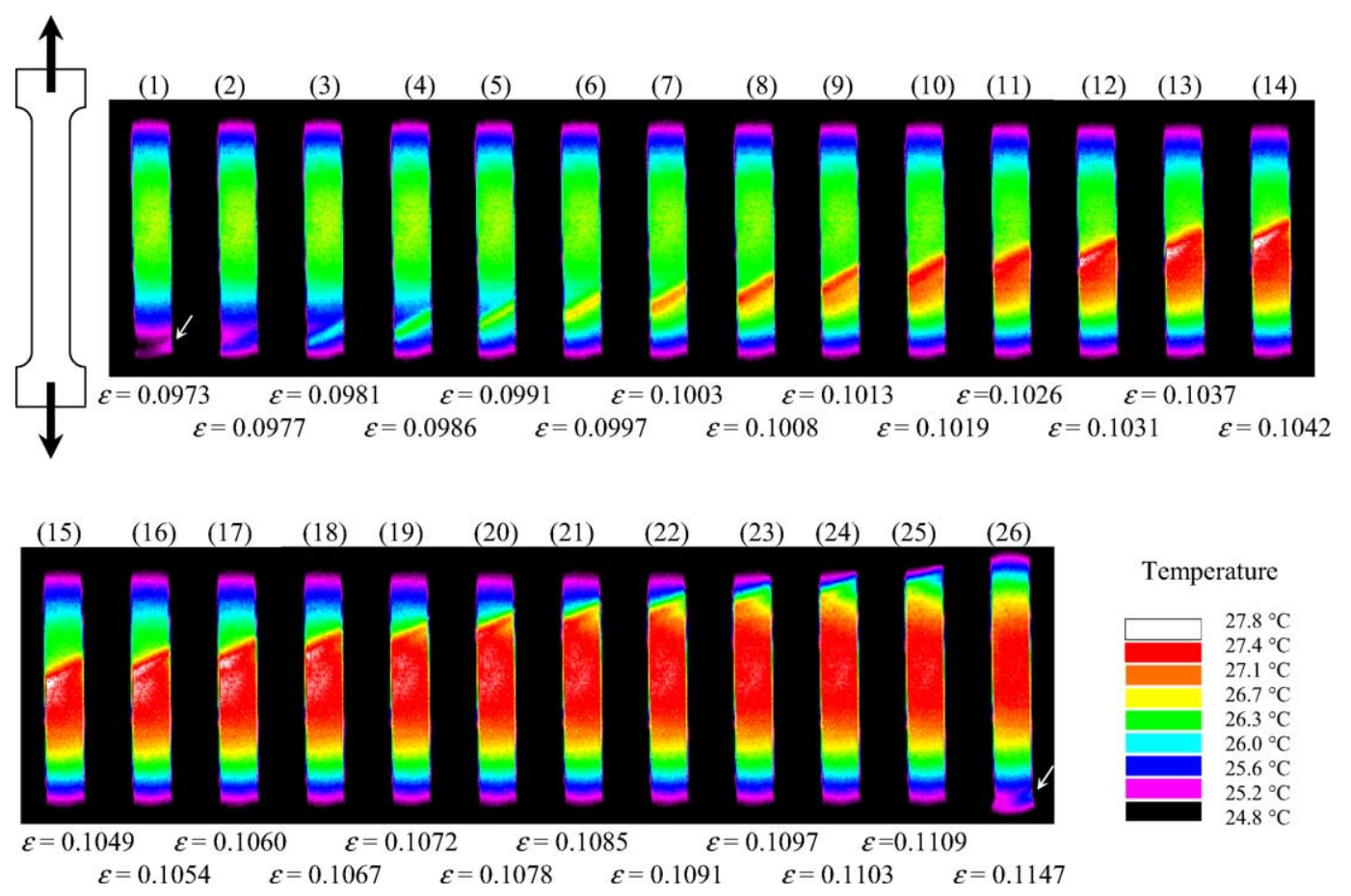

Fig. 3. Thermographs of traction specimen.

mation (Fig. 1) starts as early as the end of the elastic domain. In fact it is not necessary to reach a critical strain $\varepsilon_{\mathrm{c}}$ for the onset of the serrated yielding. The experimental determination of this critical strain is difficult because in the beginning of the tensile test the apparent propagation velocities are high and the increments of plastic deformation are very weak. The determination of the onset of the phenomenon seems strongly dependent on the precision of the experimental techniques used. As mentioned above, our measurement technique by pyrometry detects temperature variation of around $40 \mathrm{mK}$ (for a signal to noise ratio equal to 2), which corresponds to heterogeneity of the plastic deformation from approximately $0.05 \%$. However this observation about the critical strain goes against the majority of the experimental observations in literature [20,21]. Indeed, the critical strain for the onset of the serrated yielding normally increases with increas-
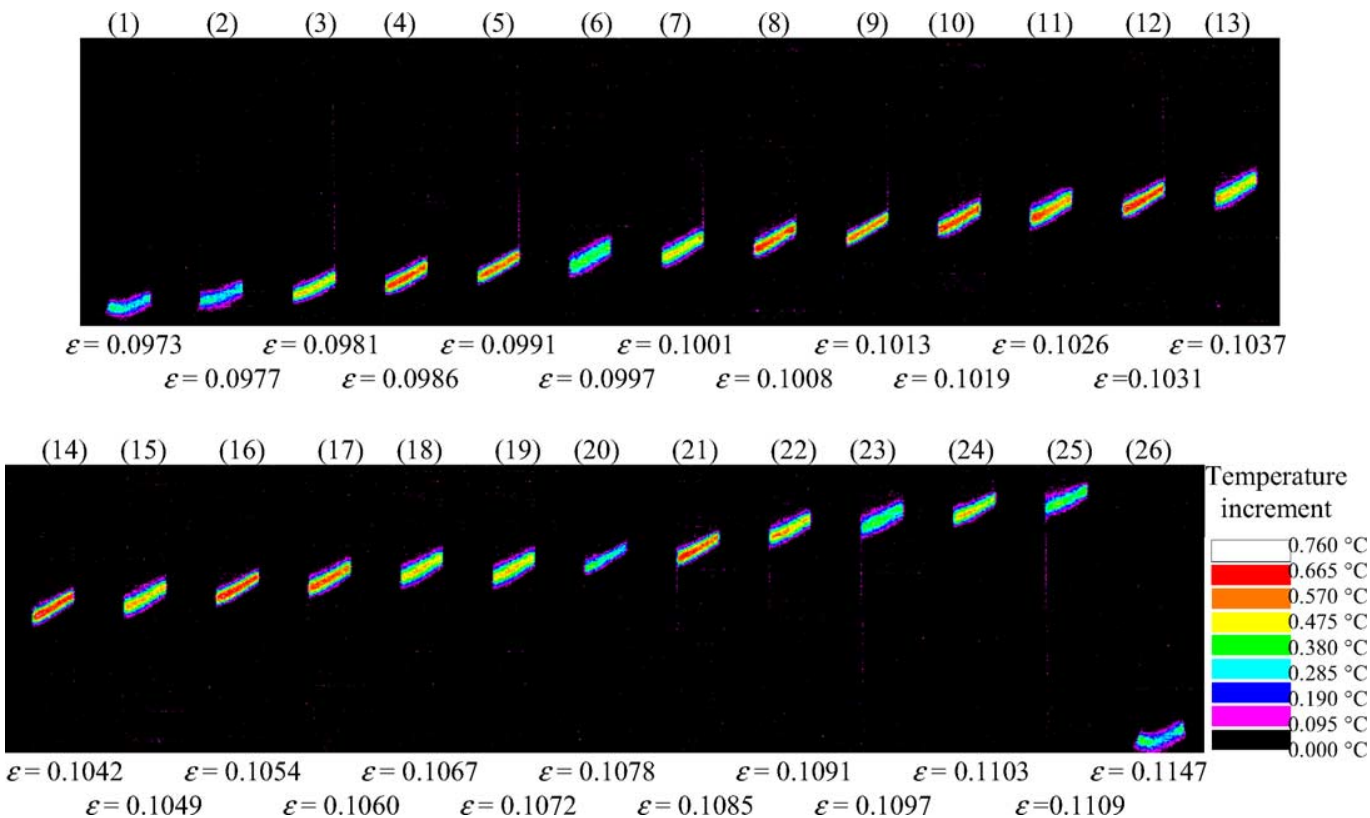

Fig. 4. Temperature increment during occurrence of PLC bands. 


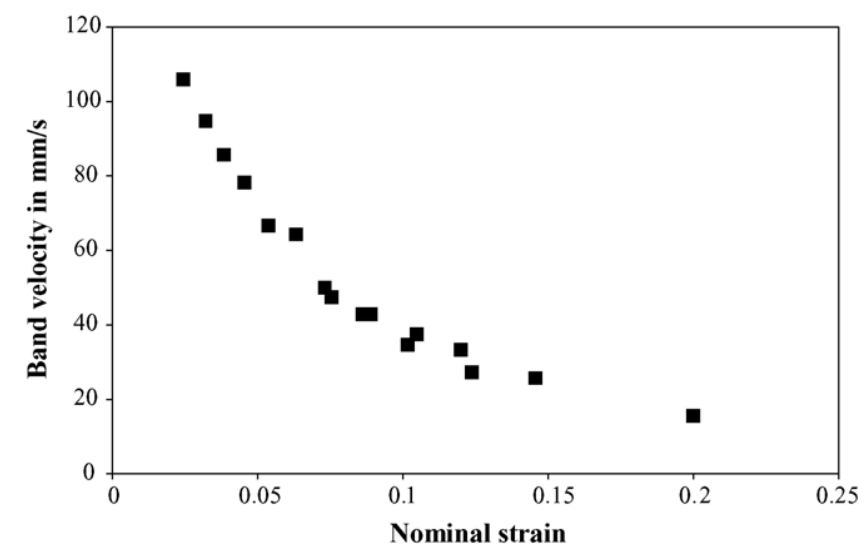

Fig. 5. Band velocity vs. total nominal strain

ing strain rate and decreases with increasing temperature. This phenomenon is called "normal" behaviour. However, the "inverse" behaviour, which is characterized by decreasing critical strain with increasing strain rate and decreasing temperature, has already been observed. Abbadi et al. [21]

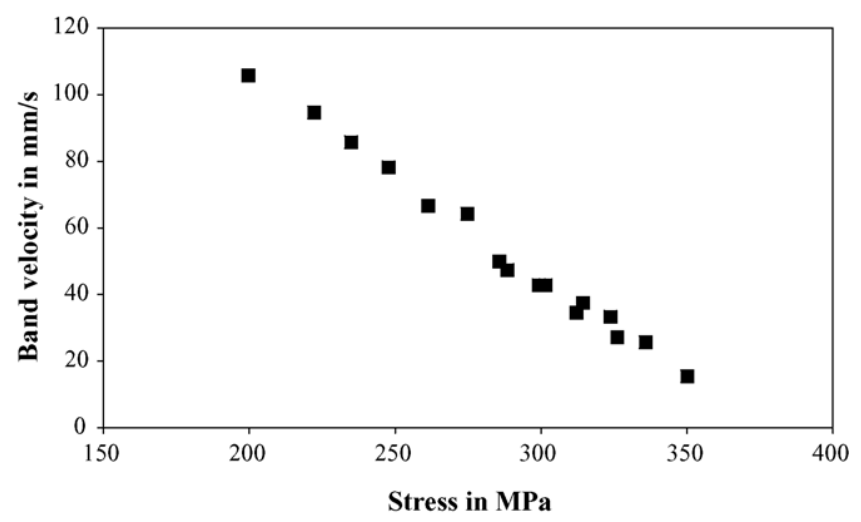

Fig. 6. Band velocity vs. stress.

have reported that in an $\mathrm{Al}-\mathrm{Mg}$ alloy, type $\mathrm{A}$ and type $\mathrm{C}$ serrations correspond respectively to the normal and the inverse behaviour and type B serrations are generally found in the transition behaviour close to the minimum value of $\varepsilon_{\mathrm{c}}$ versus strain rate. In our case, the serrations are of type B. Considering temperature measurement is a more precise method than

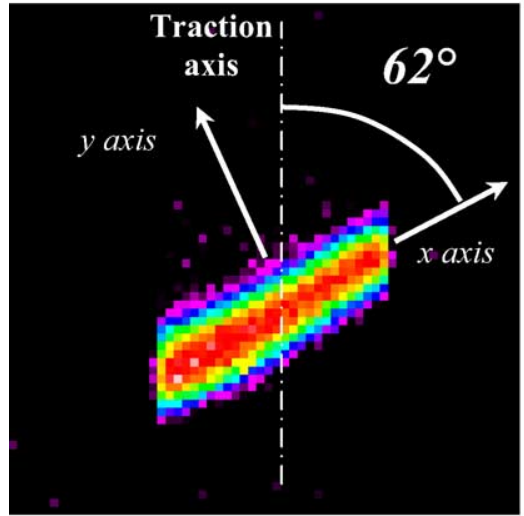

Nominal strain : $\varepsilon=0.1054$ Maximal variation of temperature : $\Delta T=0.738^{\circ} \mathrm{C}$

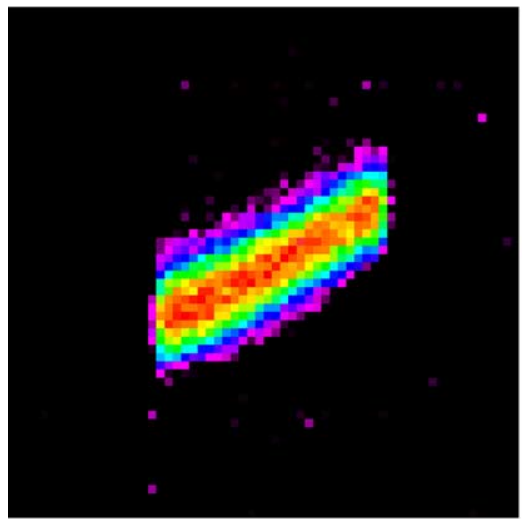

Nominal strain : $\varepsilon=0.1060$ Maximal variation of temperature : (a) $\Delta T=0.693^{\circ} \mathrm{C}$
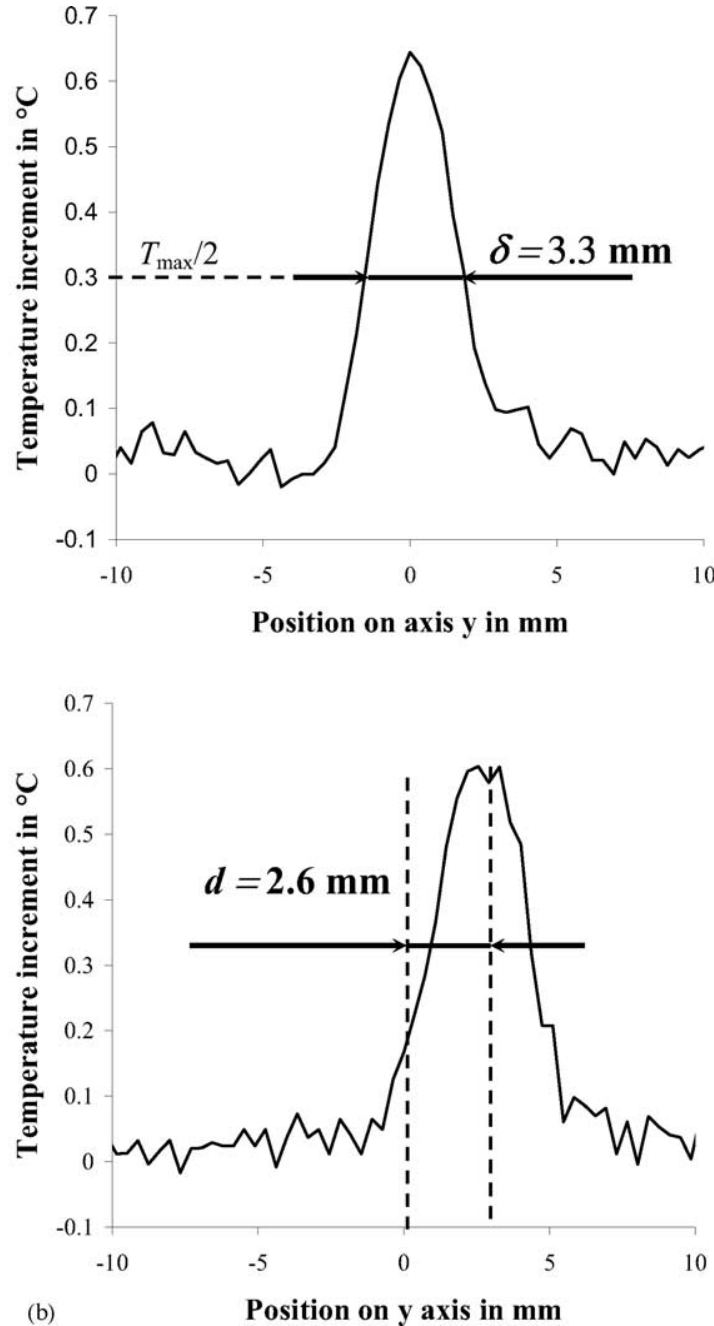

(b)

Fig. 7. (a) Band slope with the traction direction. (b) Band-width and distance between two bands. 
extensometry to visualize PLC bands and $\varepsilon_{\mathrm{c}}$ will be minimum in type B serrations, the results obtained in this study can be explained, particularly since Cuddy and Leslie [14] have reported this behaviour on an iron base metal containing $0.15 \%$ $\mathrm{Ti}$ (to remove from solution interstitial carbon, oxygen and nitrogen) with different additions of substitutional $\mathrm{Si}$. These observations confirm that enhanced diffusion due to deformation enhanced vacancies does not control the formation of serrations.

\subsection{Geometric strain heterogeneities}

Fig. 7(a) represents the temperature variations during the formation of bands 16 and 17 from Fig. 3. These pictures enable us to determine the slope angle between the bands direction and the traction axis. For frame 16 we find an angle of $62^{\circ}$. Fig. 7(b) shows the profile of the temperature variations along $y$-axis for the bands 16 and 17 . We can measure the bandwidth noted $\delta$, and is defined as the width where the temperature variation due the band-formation is higher than the maximum temperature variation divided by two. We find on frame 16 and 17 a bandwidth of respectively 3.3 and $3.6 \mathrm{~mm}$.

The assumption of an adiabatic phenomenon causes an error on the estimate of the bandwidth. The heat diffusion between the moment when the band is formed and the opening of the camera tends to overvalue the real bandwidth. Indeed, we note a small dispersion of the results. Fig. 10, given in Appendix A, shows the maximization of the estimated bandwidth. If the band is formed just after the opening of the camera, we observe, on the following picture, the heat diffusion consequences during $16.7 \mathrm{~ms}$, corresponding to the refresh time of the camera. In this most unfavourable case, the error on the bandwidth is of $1.5 \mathrm{~mm}$. The bandwidth measured on frame 16 and 17 corresponds to the finest bands among the picture 1-26.

Fig. 7(b) also allows the distance between two bands along the $y$-axis (noted $d$ ) to be measured. A distance of $2.6 \mathrm{~mm}$ is found between thermographies 16 and 17 .

We can notice that the slope of the band, the bandwidth, and the distance between two consecutive bands remain constant during the tensile test. These values are in good agreement with the few ones reported in literature [15-17].

\subsection{Middle-band time and formation-band time}

A scheme of an enlargement of the stress-strain curve is given in Fig. 8. Each serration is made of an increase of the stress, followed by an abrupt stress drop. The increase corresponds to the time between the formation of two consecutive bands called the "middle-band time" and noted $t_{\mathrm{mb}}$, while the stress drop corresponds to the sudden formation of the band. Only during this phase, does a plastic deformation occurs and represents the "formationband time" noted $t_{\mathrm{fb}}$. Then for the next serration a new single band starts ahead of the previous one, and so

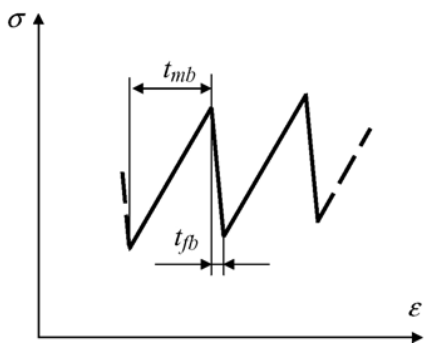

Fig. 8. Middle-band time and formation-band time in a scheme stress strain curve.

on until the upper extremity of the specimen has been reached.

We can note that the appearance of a band occurs in the majority of cases between two pictures. We can suppose that the duration of band-formation is lower than $16.7 \mathrm{~ms}$, the refresh time of the camera. In very rare cases, we can perceive the appearance of the band on two consecutive pictures. This corresponds to the particular case where the first picture shows the beginning of the band-formation and the second picture shows the completely formed band. We can thus estimate that the formation duration, which corresponds to the formation-band time, is slightly higher than the aperture time of the camera, which is $1.5 \mathrm{~ms}$. So the formation-band time is approximately a few milliseconds.

Our experimental device helps us to quantify the duration between the formation of two consecutive bands and thus to quantify the middle-band time. This waiting time evolves during the tensile test. Between the marks (a) and (b) in Fig. 1, the middle-band time is estimated at $75 \mathrm{~ms}$. At the end of the test its value is approximately $120 \mathrm{~ms}$.

\subsection{Plastic strain in the bands}

For each band, the plastic deformation increment noted $\Delta \varepsilon_{\mathrm{p}}$ could be determined from the temperature measurement and the stress level (Eq. (5)). In Fig. 9, $\Delta \varepsilon_{\mathrm{p}}$ is plotted versus the total nominal strain $\varepsilon$ during the test.

The assumption of an adiabatic phenomenon causes an error on the estimate of the increment of plastic deformation.

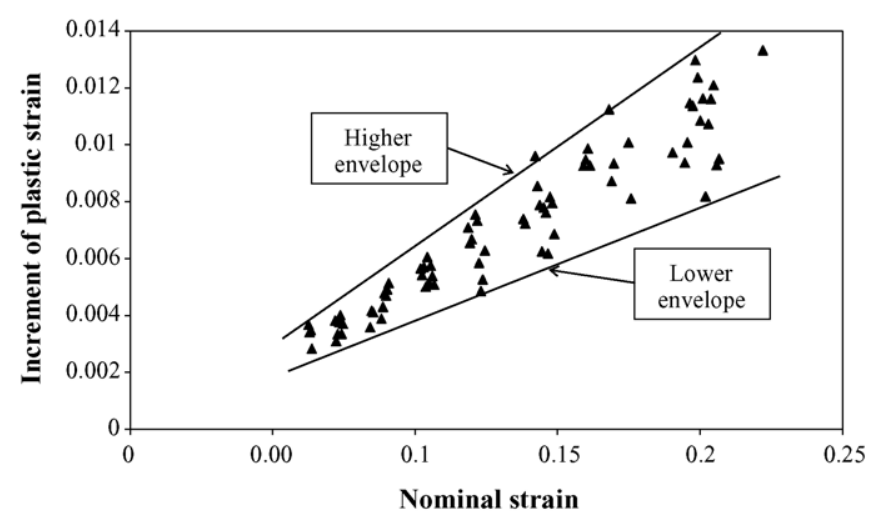

Fig. 9. Increment of plastic strain vs. nominal strain. 
The heat diffusion between the moment when the band is formed and the opening of the camera are likely to undervalue the real increment of the plastic deformation. Also, the experimental points given in Fig. 9 correspond to a minimal increment of deformation. The dispersion of the results can be explained mostly by the heat diffusion. The temperature evolution in the centre of the band versus time is given in Fig. 11, in Appendix A. The maximum relative reduction of the temperature is approximately $39 \%$ for the duration of the camera refresh time. By considering the high envelope curve in Fig. 9, we can show that the increment of plastic deformation in the band is about $7 \%$ of the total deformation (i.e. $\Delta \varepsilon_{\mathrm{p}} / \varepsilon \sim 0.07$ ).

When the band propagates, the mobile dislocation density $\rho_{\mathrm{m}}$ is related to the increment of plastic deformation $\Delta \varepsilon_{\mathrm{p}}$ by the expression:

$\rho_{\mathrm{m}}=\frac{\Delta \varepsilon}{b \delta}$

With $b$ the modulus of the Burgers vector $(0.4 \mathrm{~nm}$ for the $\mathrm{Al}$ crystal) and $\delta(3.5 \mathrm{~mm})$ the bandwidth, which is arbitrarily taken for the dislocation path. Then we obtained a mobile dislocation density of $2.9 \times 10^{5} \mathrm{~cm}^{-2}$ for the beginning of the test $(\Delta \varepsilon=0.004)$ and $9.3 \times 10^{5} \mathrm{~cm}^{-2}$ for the end of the test $(\Delta \varepsilon=0.013)$. These values are in agreement with those reported by Lacombe [15] from the studies of Korbel on $\mathrm{Cu}-\mathrm{Zn}$ alloys and by Chmelik et al. [23] from the studies of Neuhaüser. For Lacombe, $\rho_{\mathrm{m}}$ in the PLC bands will be about $10^{4} \mathrm{~cm}^{-2}$ at the deformation start and will reach $10^{6} \mathrm{~cm}^{-2}$ at the end. For Chmelik et al., each stress drop is related to a movement of at least $10^{4} \mathrm{~cm}^{-2}$ dislocations.

\section{Conclusion}

The PLC instabilities in an Al-Cu alloy were investigated by infrared pyrometry technique. In addition to the visualization of the phenomenon, this technique helps to quantify some data like geometrical characteristics and deformation increment. For the strain rate and temperature used during the tensile tests type B serrations were observed. Each stress drop corresponds to a band-formation. The band nucleation is discontinuous but regular. Each new single band starts ahead of the one preceding and so on until the opposite side of the sample. The time between two nucleations is estimated between 75 and $120 \mathrm{~ms}$ along the test. The band-formation occurring during the stress drop is very fast (a few milliseconds) and gives an increment of plastic deformation equal to $7 \%$ of the nominal deformation. The bandwidth was estimated at $3.5 \mathrm{~mm}$, and its slope with tensile axis at $62^{\circ}$. The apparent band velocity decreases during the test from 100 to $20 \mathrm{~mm} \mathrm{~s}^{-1}$.

\section{Acknowledgements}

We thank Mr. Bremond of the CEDIP Infrared System Company for the loan of the infrared camera (see http://www.cedip-infrared.com).We also thank Miss Darbord, Associated Professor at LEEE for her help on the thermal calculations.

\section{Appendix A. Thermal calculations}

We suppose that the plastic deformation is uniform in a zone of width $d=3 \mathrm{~mm}$. In this band, there is a uniform increase of the temperature. We denote the temperature of the specimen by $T_{0}$ before the band appearance and this temperature variation by $\Delta T$. This initial temperature is supposed to be uniform. The length of the band is supposed to be very large compared to its width in order to consider the unidimensional problem. We choose a $y$-axis perpendicular to the band direction. The origin of the $y$-axis is taken at the center of the band. We take $t=0$ at the time of the band creation. Only the positive values of $y$ will be considered because the problem is symmetrical compared to $x$-axis. The thermal diffusivity noted $a$ is taken equal to $9.13 \times 10^{-5} \mathrm{~m}^{2} \mathrm{~s}^{-1}$. The initial temperature is equal to $30^{\circ} \mathrm{C}$.

The resolution of the thermal problem allows to determine the temperature profile along $y$-axis according to the time. After calculation, we find the following temperature profiles:

$$
\begin{aligned}
& \text { For } 0 \leq y \leq \frac{d}{2}: \quad T(y, t)=T_{0}+\Delta T \\
& \qquad \begin{array}{l}
-\frac{\Delta T}{2}\left(\operatorname{erfc}\left(\frac{d / 2+y}{2 \sqrt{a t}}\right)+\operatorname{erfc}\left(\frac{d / 2-y}{2 \sqrt{a t}}\right)\right) \\
\text { For } y \geq \frac{d}{2}: \quad T(y, t) \\
\quad=T_{0}+\frac{\Delta T}{2}\left(\operatorname{erfc}\left(\frac{y-d / 2}{2 \sqrt{a t}}\right)-\operatorname{erfc}\left(\frac{y+d / 2}{2 \sqrt{a t}}\right)\right)
\end{array}
\end{aligned}
$$

The temperature evolution is represented in Fig. 10 for time $t=0, t=8.3 \mathrm{~ms}$ and $t=16.7 \mathrm{~ms}$. At $t=8.3 \mathrm{~ms}$ and $t=16.7 \mathrm{~ms}$ the bandwidth is 3.6 and $4.5 \mathrm{~mm}$, respectively. These values correspond to a relative variation of 20 and $53 \%$, respectively.

The temperature evolution in the center of the band $(y=0)$ can also be expressed:

$$
T(t)=T_{0}+\Delta T \cdot \operatorname{erf}\left(\frac{d / 2}{2 \sqrt{a t}}\right)
$$

and the relative variation of temperature is:

$$
\frac{T(t)-T_{\max }}{T_{0}-T_{\max }}=\operatorname{erfc}\left(\frac{d / 2}{2 \sqrt{a t}}\right)
$$




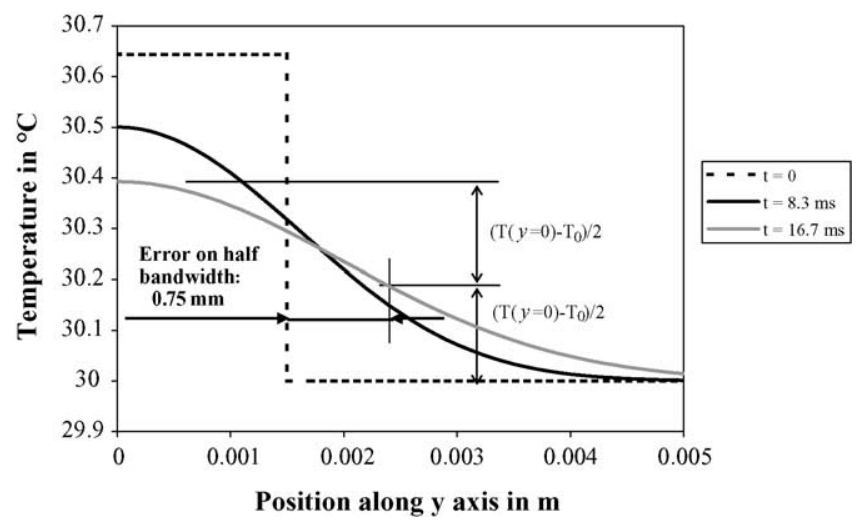

Fig. 10. Temperature profile in the specimen.

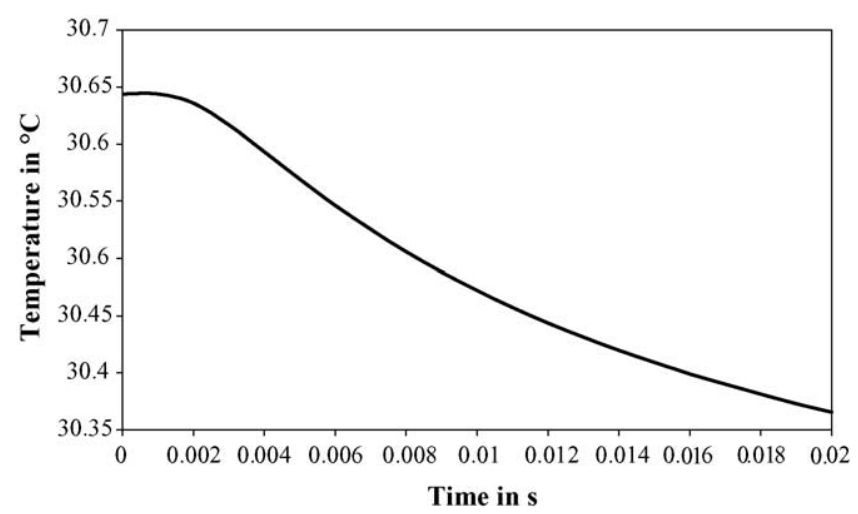

Fig. 11. Temperature evolution in the center of the band vs. time.

where $T_{\max }$ is the initial temperature in the center of the band. The temperature evolution in the center of the band is shown as a function of time in Fig. 11.

\section{References}

[1] J.D. Baird, Iron Steels (1963) 186, 326, 368, 400, 450.

[2] Y. Estrin, L.P. Kubin, in: H.B. Mühlhaus (Ed.), Continuum Models for Materials with Microstructure, Wiley, New York, 1995, p. 395.
[3] J.D. Baird, Dynamic strain aging, in: A.S.M. International (Ed.), The Inhomogeneity of Plastic Deformation, Ohio, Metals Park, 1973, p. 191.

[4] A.S. Keh, Y. Nakada, W.C. Leslie, in: A.R. Rosenfeld (Ed.), Dislocation Dynamics, McGraw-Hill, New York, 1968, p. 381.

[5] D. Wagner, J.C. Moreno, C. Prioul, Rev. Met. 12 (2000) 1481.

[6] L.P. Kubin, Y. Estrin, J. Phys. III 1 (1991) 929.

[7] H. Neuhäuser, C. Schwink, in: R.W. Cahn, P. Haasen, Kramer (Eds.) Mater. Sci. Technol. 6 (1993).

[8] A.H. Cottrell, Trans. Met. Soc. AIME 212 (1953) 192.

[9] P.G. McCormick, Acta Metall. 20 (1972) 35.

[10] A. Van den Beukel, Phys. Stat. Sol. 30 (1975) 197.

[11] A.W. Sleeswijk, Acta Metall. 6 (1958) 598.

[12] R.A. Mulford, U.F. Kocks, Acta Metall. 27 (1979) 1125.

[13] B.J. Brindley, P.J. Worthington, Metall. Rev. 15 (1970) 101.

[14] L.J. Cuddy, W.C. Leslie, Acta Metall. Mater. 20 (1972) 1157.

[15] P. Lacombe, Matériaux et Techniques 8-9 (1985) E5.

[16] P. Rodriguez, in: R.W. Cahn (Ed.), Encyclopedia of Materials Science and Engineering-Supplementary, vol. 1, Pergamon, 1988, p. 504.

[17] P.G. McCormick, S. Venkadesan, C.P. Ling, Scripta Metall. Mater. 29 (1993) 1159.

[18] M. Lebyodkin, L. Dunin-Barkowskii, Y. Bréchet, Y. Estrin, L.P. Kubin, Acta Metall. Mater. 48 (2000) 2529.

[19] J.L. Strudel, in: P. Groh, L.P. Kubin, J.L. Martin (Eds.), Dislocations et déformation plastique, Yravals, Les éditions de la physique, Paris, 1979.

[20] J. Balik, P. Lukac, Acta Metall. Mater. 41 (1993) 1447.

[21] M. Abbadi, P. Hähner, A. Zeghloul, Mater. Sci. Eng. A 337 (2002) 194.

[22] Y. Bréchet, Y. Estrin, Acta Metall. Mater. 43 (1995) 955.

[23] F. Chmelik, A. Ziegenbein, H. Neuhäuser, P. Lukac, Mater. Sci. Eng. A 324 (2002) 200.

[24] R. Shabadi, S. Kumar, H.J. Roven, E.S. Dwarakadasa, Mater. Sci. Eng. A 364 (2004) 140.

[25] A. Chrysochoos, H. Louche, Int. J. Eng. Sci. 38 (2000) 1759.

[26] M. Zhou, A.J. Rosakis, G. Ravichandran, J. Mech. Phys. Sol. 44 (1996) 981.

[27] P.R. Guduru, A.T. Zehnder, A.J. Rosakis, G. Ravichandran, Eng. Fract. Mech. 68 (2001) 1535.

[28] N. Ranc, V. Pina, P. Hervé, L. Taravella, P.F. Louvigné, J. Phys. IV 110 (2003) 417.

[29] G. Sutter, L. Faure, A. Molinari, N. Ranc, V. Pina, Int. J. Mach. Tools Manufac. 43 (2003) 671.

[30] W. Thomson, Trans. Roy. Soc. 20 (1853) 261.

[31] G.I. Taylor, H. Quiney, Proc. Roy. Soc. A143 (1934) 307. 